\title{
Through-Wafer Electrical Interconnects Compatible With Standard Semiconductor Processing
}

\author{
Eugene M. Chow, Aaron Partridge, Calvin F. Quate, and Thomas W. Kenny \\ Ginzton Laboratory, Stanford University \\ Stanford, CA, 94305-4085
}

\begin{abstract}
The fabrication and characterization of high-density electrical through-wafer interconnects on silicon substrates are described. A $20 \mu \mathrm{m}$ via is etched with a high-density plasma, followed by conformal oxide deposition, polysilicon deposition, and diffusion doping. The process uses high-temperature compatible materials and results in a filled via, making it suitable for standard semiconductor processing and wafer-handling equipment. Resistance $(14 \Omega)$, capacitance $(0.6 \mathrm{pF})$ and noise measurements are presented.
\end{abstract}

\section{INTRODUCTION}

Electrical through-wafer interconnects (ETWI) in silicon have significant applications for integrated circuits (IC) and microelectrical mechanical systems (MEMS). New electrical structures are enabled, such as high-Q three-dimensional inductors or vertical diodes for charged particle detection [1-3]. Stacks of IC or sensor chips are possible, saving space, and adding packaging and system design flexibility [4]. If the ETWI are small and densely packed, they could enable the transfer of thousands of signals between stacked chips. Small ETWI benefit arrayed detectors, such as ultrasound imagers, and minimize non-sensing area and thus enhance performance [5]. In previous work on twodimensional arrays of scanning probes, we demonstrated how small ETWI $(30 \mu \mathrm{m})$ solve the geometric problem of aligning arrays of micron-sized tips to arbitrary planar samples in the presence of wire-bonds $[6,7]$. ETWI can provide a solution to MEMS-IC integration problems, as they permit MEMS and ICs to be fabricated on separate wafers, and afterwards be bonded together to form a system [5].

Unfortunately, previous interconnects use techniques which are not generally compatible with standard semiconductor processing. Laser drilling can make $20 \mu \mathrm{m}$ diameter holes, but the process is serial and not as geometrically flexible as standardized lithography and etching techniques [2]. A wet etch is most common, leaving large holes in the wafer $(>200 \mu \mathrm{m})$, precluding future vacuum handling and seriously impeding the use of many etching, metalization, and lithography tools $[8,9]$. Subsequent layers often require unconventional lithography, such as electrodeposited resist or shadow masks $[1,10]$. High-density anisotropic plasma etching is attractive for via formation, as it enables smaller holes than wet etching and more flexible geometry. While we previously plasma etched a small $(30 \mu \mathrm{m})$ via and used spin-on thick resist patterning, we could not use vacuum handling because the interconnects were not filled [7].

Our primary ETWI application is for integration with micromachined scanning probe arrays, for applications in imaging, data storage, and lithography [11-13]. These sensors are delicate, freestanding structures, so ETWI prior to device fabrication is preferred. Sensor packing density, and thus small ETWI, are critical. High-temperature thin film steps are necessary for subsequent device fabrication, so ETWI fabrication precludes the use of metals. This is acceptable because resistances of only tens of ohms are required, as they are in series with kilo-ohm piezoresistors. Signals are generally in the kilohertz range, so picofarad capacitances are more than adequate. To not degrade sensor sensitivity, noise smaller than the piezoresistor cantilever noise is desired.

In this work we have fabricated and characterized ETWI which meet these criteria. They can be integrated with subsequent standard semiconductor processing, as the interconnects are filled and use only standard high-temperature compatible materials.

\section{FABRICATION}

The basic fabrication process (Fig. 1), consists of etching a high-aspect ratio through-wafer via and subsequently coating with an insulator and then a conducting signal layer.

a)

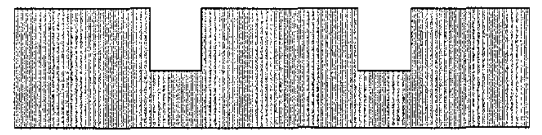

b)
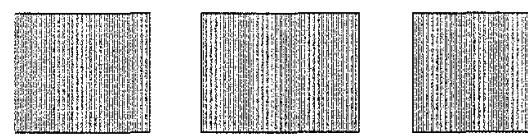

c)
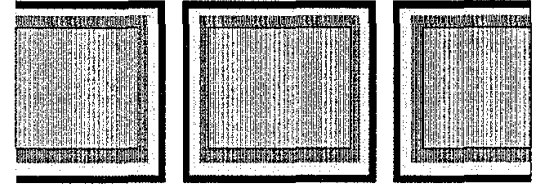

d)

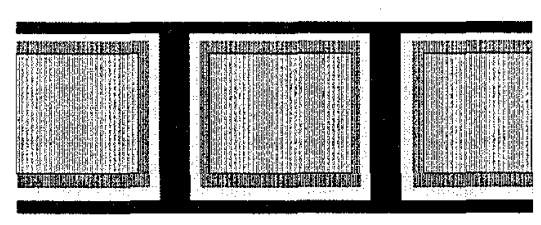

e)

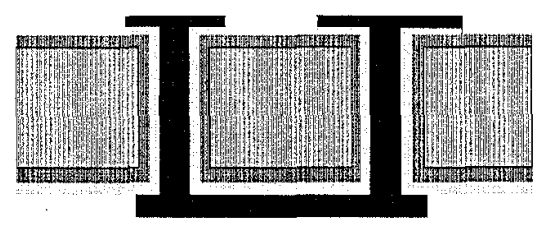

Figure 1. Fabrication process for a pre-process through-wafer interconnect. a) Holes are etched halfway through the wafer. b) Holes are etched from the backside of the wafer, meeting the frontside holes. c) After diffusion doping, a ground plane oxide and then polysilicon are deposited. d) The polysilicon is diffusion doped, and additional polysilicon is deposited to fill. More diffusion and an anneal follows. e) The conduction layer is patterned on both sides. 
Etching the vias is the first, and most critical step. Ten micron thick photoresist (Shipley SPR220-7) is initially coated on one side of the wafer. After a 120 second hotplate bake at $90^{\circ} \mathrm{C}$, the other side is similarly coated, and the wafer is oven baked at $110^{\circ} \mathrm{C}$ for 60 minutes. Previously patterned alignment marks on the front side silicon, combined with a backside contact mask aligner (KarlSuss), enable the exposure of aligned $20 \mu \mathrm{m}$ circles on both sides of the wafer. The wafers then sit in air for 8 hours to absorb water. This moisture helps to ensure shorter develop times (90 seconds in Shipley LDD26W) and thus straighter photoresist sidewalls.

A high-density plasma etch (Surface Technology Systems) is performed from the front side of the wafer for 210 minutes. The etcher uses the Bosch etching process, switching hetween sequential passivation $\left(85 \mathrm{sccm} \mathrm{C}_{4} \mathrm{~F}_{8}, 6\right.$ sec., $0 \mathrm{~W}$ platen, $600 \mathrm{~W}$ coil, 16 mtorr), and etching ( $80 \mathrm{sccm} \mathrm{SF}_{6}, 8 \mathrm{sec}$., $8 \mathrm{~W}$ platen, 600 W coil, 33 mtorr). Resist is then coated and baked on the front side for use as a seal from the wafer-chuck helium gas cooling. This is necessary because another 180 minute etch, this time from the backside, is then performed to punch through the via. The etch is very anisotropic, and alignment is as accurate as the lithography $(+/-1 \mu \mathrm{m})$. The diameter varies from $24 \mu \mathrm{m}$ (top/bottom) to 18 $\mu \mathrm{m}$ (middle). By etching from both sides of the wafer, the required aspect ratio is reduced by two from $40: 1$ to $20: 1$, and it is possible to etch a $20 \mu \mathrm{m}$ circle through a $400 \mu \mathrm{m}$ thick wafer. The overetch time of the second etch, which punches through, should be minimized to reduce lateral etching of the front side silicon. Using an etch with increased passivation for the final 30 minutes helps to limit this effect. Figure 2 depicts an etched via

Etches with the required aspect ratios $(>20: 1)$ have been previously reported, but they are of trenches, with one dimension very long $(\sim 1000 \mu \mathrm{m})$, or not as deep as through a $400 \mu \mathrm{m}$ thick wafer $[3,14,15]$. Decreased etching rate for small areas, characteristic for flourinc-based plasmas, is particularly severe for deep etches [16]. We measured the time to etch trenches $(20 \mu \mathrm{m} x$ $1000 \mu \mathrm{m}) 200 \mu \mathrm{m}$ deep to be three times less than the time to etch a circle ( $20 \mu \mathrm{m}$ diameter) to the same depth. By etching from both sides of the wafer, the average etch rate is higher, which shortens the total etch time and permits the use of thinner photoresists.

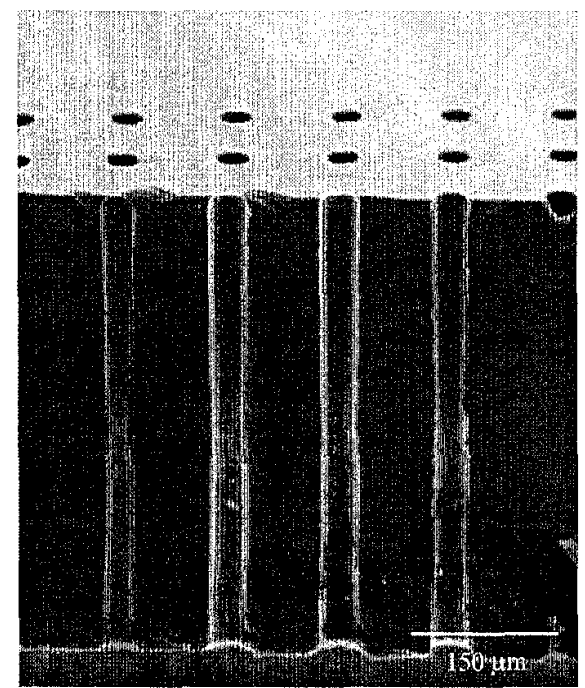

Figure 2. SEM cross-section after plasma etching vias through the wafer.
After forming the via, conformal thin films are deposited. To shield the signal, a ground plane is formed by diffusion doping the wafer and vias with phosphorous for 30 minutes at $1000^{\circ} \mathrm{C}$. This is important if the ETWI is used to conduct small signals from a sensor to an amplifier, as it shields electrical noise from the environment. The diffusion forms a glass which is removed by a brief etch in buffered hydrofluoric acid (BHF). There is a possibility the BHF does not fully wet all of the vias, even with surfactant and agitation. This could possibly leave unwanted interface charges which would compromise the capacitance stability under different biases. The ground shield masks such effects if present.

For electrical isolation, thermal silicon dioxide $(2 \mu \mathrm{m})$ is grown at $1100^{\circ} \mathrm{C}$. For signal conduction, low pressure chemical vapor deposition (LPCVD) polysilicon $(3 \mu \mathrm{m})$ is deposited at 600 ${ }^{\circ} \mathrm{C}$, followed by phosphorous diffusion at $1000^{\circ} \mathrm{C}$ for 30 minutes, and its corresponding BHF dip. To fill the via, more LPCVD polysilicon is deposited $(7 \mu \mathrm{m})$. Another 30 minute phosphorous diffusion is performed to make the surface conducting, followed by another BHF dip, and then a 120 minute anneal at $1000^{\circ} \mathrm{C}$ to fully drive dopants throughout the polysilicon. Conventional spin-on resist is then used to pattern the polysilicon with $\mathrm{SF}_{6}$ plasma, repeating on each side to complete the ETWI (Fig. 3).

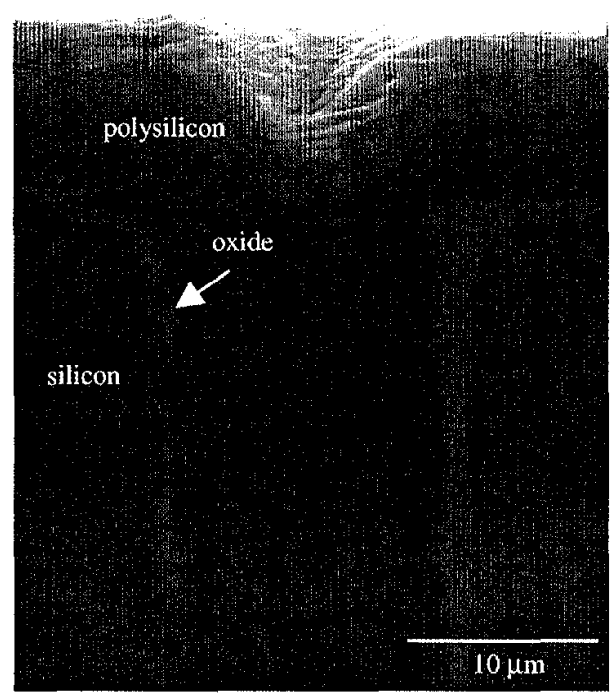

Figure 3. SEM cross-section of a completed through-wafer interconnect. Planarity over the via is adequate for conventional spin-on resist lithography. Polishing can be applied if improved planarization is required.

\section{CHARACTERIZATION}

Current versus voltage measurements were made of interconnect chains to determine the resistance of the structures and demonstrate ohmic behavior (Fig. 4). The slope of each curve is inversely proportional to the resistance sum of the ETWI and the planar lines between them ( $50 \mu \mathrm{m}$ wide, $110 \mu \mathrm{m}$ for this device). A slight hyperbolic sine form is observed for smaller resistance ETWI, characteristic of polysilicon resistors [17]. Poor probing contact or Joule heating could also cause such nonlinearites [18]. A small Schottky diode formed by the electrical probe station tip and the polysilicon resistor contact pad would have a pronounced effect on small resistance structures, where the diode effective 
resistance is comparable to that of the ETWI. Joule heating effects would also be pronounced for lower resistance. Measurements were repeated multiple times and slopes (resistances) were found to vary $+1-5 \%$

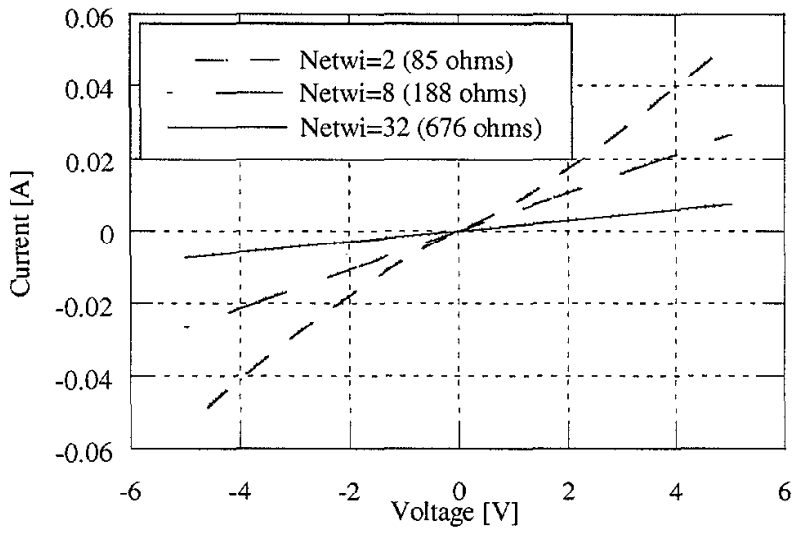

Figure 4. Current versus voltage for consecutive chains of 2,8 , and 32 ETWI

'To extract the resistance of an individual ETWI, planar test structures (no ETWI), were similarly measured. These resistances were subtracted from the chain resistances and plotted by number of ETWI in the chain (Fig. 5). The slope of the resulting line gives an average single ETWI resistance of $14 \Omega$. Bulk model calculations give similar results, as a cylindrical wire has a resistance of $R=\rho L / A$, where $\rho$ is the polysilicon resistivity, $L$ the length of the ETWI, $A=\pi r_{c}^{2}$ the cross sectional area, and $r_{c}$ the radius of the conducting core. Four point probe measurements were performed to obtain a doped polysilicon sheet resistance $R_{s}=$ $\rho / t$ of $1.1 \Omega / s q$., where $t$ is the film thickness. Assuming the via was not significantly overfilled with polysilicon, then $t=r_{c}$. For $L$ $=415 \mu \mathrm{m}$ and an approximate $r_{c}=10 \mu \mathrm{m}$, this gives $R=14.5 \Omega$.

Capacitance was similarly measured on chains of interconnects. Test structures were measured so that the capacitance due to the planar lines between the ETWI could be subtracted. Capacitance was measured between the ground plane and the signal plane at $10 \mathrm{kHz}$, with the polysilicon-oxide-silicon structure biased in accumulation. Capacitance measurements for a single ETWI chain were repeatable to within $3 \%$. The slope of a fitted line (Fig. 5) results in a measured $0.6 \mathrm{pF}$ per ETWI. We model the capacitance as a coaxial transmission, where $\mathrm{C}=2 \pi \varepsilon_{o x}$

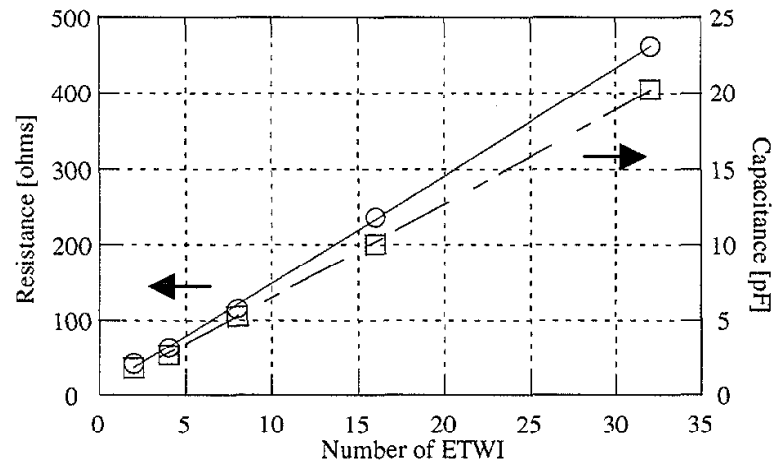

Figure 5. Resistance and capacitance of ETWI chains. $\varepsilon_{o} L / \ln \left(r_{c} / r_{v}\right)$, and $r_{v}$ is the outer conductor dianeter (the via diameter after oxidation), $\varepsilon_{o x}$ the relative dielectric permitivity of silicon dioxide, and $\varepsilon_{o}$ the diclectric permitivity of free space. Taking $r_{v}=12 \mu \mathrm{m}$ and $\varepsilon_{o x}=3.9$ gives a capacitance of $0.5 \mathrm{pF}$ per ETWI.

Noise characteristics were measured by placing the ETWI chains in a matched Wheatstone bridge. A chain of 128 ETWI was compared to a metal film resistor, both with a resistance of $2100 \Omega$. The ETWI shows increased 1/f noise compared to the reference resistors. The intersection of the $1 / \mathrm{f}$ noise with the Johnson noise limit is extrapolated to be $\sim 10 \mathrm{kHz}$, similar to previous work [17, 18]. Both spectra are limited by the noise floor $(8 \mathrm{nV} /$ sqri $\mathrm{Hz})$ of the amplifier (AD624 instrumentation amplifier at $100 \times$ gain), which exceeds the Johnson noise of a $2100 \Omega$ resistor $(3 \mathrm{nV} /$ sqrt $\mathrm{Hz}$ ). A typical spectrum is given in Figure 6.

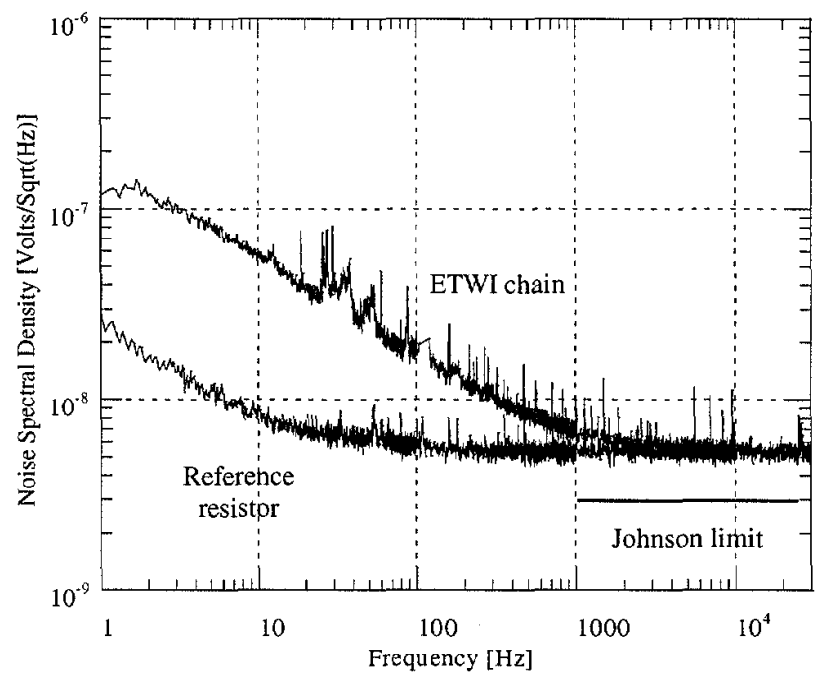

Figure 6. Noise spectrum for ETWI chains compared to a reference resistor:

\section{DISCUSSION}

The resistance and capacitance of the ETWI are satisfactory for use our standard piezoresistor sensors. The slight nonlinearity in the current-voltage curve changes the effective resistance for large voltage swings. Since piezoresistive signals are generally small $(<1 \mathrm{mV})$ and centered around a constant bias, this is acceptable for our applications. A capacitance of $\sim 1 \mathrm{pF}$, with a 14 $\Omega$ resistance, gives a time constant of $10^{-11}$ seconds, significantly more than adequale for most piezoresistive applications. The measured low frequency noise of a ETWI chain appears to be comparable to a typical piezoresistive cantilever, which would degrade cantilever sensitivity [19]. This needs to be investigated more by measuring individual ETWI or ETWI in series with piezoresistor sensors. Aluminum contact pads could help with possible contact problems.

Thermal cycling, stress, and reliability issues have not yet been studied for this scale geometry. However, smaller polysilicon contact plugs (few microns deep) with similar or greater aspect ratios have been successfully integrated with standard IC processes $[20,21]$. The thermal strain mismatch between silicon and polysilicon is small, so stresses are expected to be from oxide mismatches, and polysilicon internal stress [22]. An occasional ETWI exhibited a filling void, but because they were sealed under 
vacuum we do not expect subsequent stressing in low-pressure systems. Possible absolute resistance variations because of voids are acceptable for this application.

Variations on the ground plane technique are being considered. The current technique leaves the device surface heavily doped and thus unfit for piezoresistor formation. One solution is to not use a ground plane, which might be acceptable for certain applications. While this decreases the total ETWI capacitance, because of the bulk silicon depletion, the oxide thickness still sets the maximum capacitance for the ETWI because the two capacitors are in series. Another approach is to keep the ground plane, but first deposit an isolating oxide and then doped polysilicon ground plane. After this, the insulating oxide is deposited, followed by the signal layer. This has the added advantage of reducing the overall ETWI capacitance (proportional to the signal conduction layer radius), without requiring higher aspect-ratio etching.

\section{CONCLUSIONS}

Through-wafer electrical interconnects which can be integrated with standard semiconductor processing have been demonstrated. The small size ( $20 \mu \mathrm{m}$ diameter) makes the ETWI attractive for high-density applications. Unlike previous work, the ETWI are filled and uses high-temperature compatible materials, making them compatible with standard vacuum handling equipment and subsequent integrated circuit processing. The resistance $(14 \Omega)$ is adequate for piezoresistive applications, where the series sensor resistor is typically $>1 \mathrm{k} \Omega$. The low capacitance $(<1 \mathrm{pF})$ is attractive for high-speed applications. The challenge of combining MEMS with IC electronics is one of many applications this technology helps to address.

\section{ACKNOWLEDGMENTS}

We wish to thank Dr. H.T. Soh, C.H. Cheng, X.C. Jin, B.T. Khuri-Yakub, and S. Calmes for valuable discussions and advice. This work is supported by JSEP and NSF, and makes use of the National Nanofabrication Users Network facilities funded by NSF (ECS-9731294).

\section{REFERENCES}

1. H. T. Soh, C. P. Yue, A. McCarthy, et al., "Ultra-low resistance, through-wafer via (TWV) technology and its applications in three dimensional structures on silicon", Japanese Journal of Applied Physics, Part 1 (Regular Papers, Short Notes \& Review Papers), 38(4B) 2393-6 (1999).

2. T. R. Anthony, "Forming electrical interconnections through semiconductor wafers", Journal of Applied Physics, 52(8) 5340-9 (1981).

3. C. Kenney, S. Parker, J. Segal, et al., "Silicon detectors with 3-D electrode arrays: fabrication and initial test results", IEEE Transactions on Nuclear Science, 46(4, pt.3) 1224-36 (1999).

4. M. Heschel, J. F. Kuhmann, S. Bouwstra, et al., "Stacking technology for a space constrained microsystem", Journal of Intelligent Material Systems and Structures, 9(9) 749-54 (1998).

5. X. C. Jin, S. Calmes, C. H. Cheng, et al. "Micromachined capacitive ultrasonic immersion transducer array", 10th International Conference on Solid-State Sensors and Actuators (Transducers 99), Sendai, Japan, 6/710/99, The Institute of Electrical Engineers of Japan (1999), pp. 15001503
6. E. M. Chow, H. T. Soh, H. C. Lee, et al., "Integration of through-wafer interconnects with a two-dimensional cantilever array", Sensors and Actuators, Physical A. (Accepted for publication in Vol, 82-83).

7. E. M. Chow, H. T. Soh, H. C. Lee, et al. "Two-dimensional cantilever arrays with through-wafer interconnects", The 10th International Conference on Solid-State Sensors and Actuators, Sendai, Japan, 6/710/99, Institute of Electrical Engineers of Japan (1999), pp. 1886-1887.

8. C. Christensen, P. Kersten, S. Henke, et al., "Wafer through-hole interconnections with high vertical wiring densities", IEEE Transactions on Components, Packaging, and Manufacturing Technology, Part A, 19(4) 516-22 (1996)

9. S. Linder, H. Baltes, F. Gnaedinger, et al. "Fabrication technology for waler through-hole interconnections and three-dimensional stacks of chips and wafers", Proceedings IEEE Micro Electro Mechanical Systems. An Investigation of Micro Structures, Sensors, Actuators, Machines and Robotic Systems (Cat. No.94CH3404-1), New York, NY, USA, IEEE (1994), pp. 349-354.

10. G. J. Burger, E. J. T. Smulders, J. W. Berenschot, et al., "Highresolution shadow-mask patterning in deep holes and its application to an electrical wafer feed-through", Sensors and Actuators A-Physical, 54(1-3) 669-673 (1996).

11. S. C. Minne, S. R. Manalis, and C. F. Quate, "Parallel atomic force microscopy using cantilevers with integrated piezoresistive sensors and integrated piezoelectric actuators", Applied Physics Letters, 67(26) 3918 20 (1995).

12. M. Lutwyche, C. Andreoli, G. Binnig, et al., " $5 \times 5$ 2D AFM cantilever arrays a first step towards a Terabit storage device", Sens. Actuators A, Phys. (Switzerland), Sensors and Actuators A (Physical), A73(1-2) 89-94 (1999).

13. K. Wilder and C. F. Quate, "Scanning probe lithography using a cantilever with integrated transistor for on-chip control of the exposing current", J. Vac. Sci. Technol. B, Microelectron. Nanometer Struct. (USA), Journal of Vacuum Science \& Technology $B$ (Microelectronics and Nanometer Structures), 17(6) 3256-61 (1999).

14. A. A. Ayon, R. Braff, C. C. Lin, et al., "Characterization of a time multiplexed inductively coupled plasma etcher", Joumal of the Electrochemical Society, 146(1) 339-49 (1999).

15. J. Bhardwaj, H. Ashraf, A. McQuarrie, et al. "Dry silicon etching for MEMS", Proceedings of the Third International Symposium on Microstructures and Microfabricated Systems, Pennington, NJ, USA, Electrochem. Soc (1997), pp. 118-130.

16. K. A. Gottscho, C. W. Jurgensen, and D. J. Vitkavage, "Microscopic uniformity in plasma etching", Journal of Vacuum Science \& Technology $B$ (Microelectronics and Nanometer Structures), 10(5) 2133-2147 (1992).

17. M. Y. Luo and G. Bosman, "An analytical model for $1 / \mathrm{f}$ noise in polycrystalline silicon thin films", IEEE Transactions on Electron Devices, 37(3, pt.1) 768-74 (1990).

18. M. J. Deen, S. Rumyantsev, and J. Orchard-Webb, "Low frequency noise in heavily doped polysilicon thin film resistors", Journal of Vacuum Science \& Technology $B$ (Microelectronics and Nanometer Structures), 16(4) 1881-4 (1998).

19. B. W. Chui, T. D. Stowe, J. Yongho Sungtaek, et al., "Low-stiffness silicon cantilevers with integrated heaters and piezoresistive sensors for high-density AFM thermomechanical data storage", Journal of Microelectromechanical Systems, 7(1) 69-78 (1998).

20. J. M. Drynan, E. Ikawa, and T. Kikkawa. "Fabrication of polysilicon plugs for deep-submicron contact-holes", 1990 Proceedings. Seventh International IEEE VLSI Multilevel Interconnection Conference (Cat. No.90TH0325-1), New York, NY, USA, IEEE (1990), pp. 494.

21. T. Hamajima and Y. Sugano. "Low contact resistance polysilicon plug for halfmicron CMOS technology", 1989 Proceedings. Sixih International IEEE VLSI Multilevel Interconnection Conference (Cat. No.89TH0259-2), New York, NY, USA, IEEE (1989), pp. 508.

22. D. Chidambarrao, J. P. Peng, and G. R. Srinivasa, "Stresses in silicon substrates near isolation trenches", Journal of Applied Physics, 70(9) 4816-22 (1991). 